\title{
Artritis séptica de rodilla por Staphylococcus warneri
}

\author{
Rodríguez-Montserrat D,* Calle-García JA, ${ }^{* *}$ Dellonder-Frigolé J,** Isernia V,*** Molinos-Abdós S, ${ }^{* * * *}$ \\ Hernández-Hermoso JA,***** Martínez-Pastor JC******
}

Hospital Universitario «Germans Trias i Pujol». Barcelona

RESUMEN. La artritis séptica suele presentarse como un proceso articular agudo que puede provocar una rápida destrucción del cartílago, si no se toman las medidas terapéuticas necesarias. De manera poco frecuente, el Staphylococcus warneri puede ser la causa de esta patología aunque por su dificultad diagnóstica podemos cometer errores en su tratamiento. Presentamos el caso de un paciente con artritis séptica de rodilla por este germen y pretendemos remarcar cuáles son las medidas diagnósticas y recomendaciones a tener en cuenta para esta infección osteoarticular.

Palabras clave: Artritis séptica, rodilla, diagnóstico, infección.

\section{Introducción}

El Staphylococcus warneri es, junto al Staphylococcus epidermidis, uno de los más frecuentes estafilococos coagulasa negativos que se encuentran en la piel de los humanos. Otras localizaciones como saprófito, además de la piel, son la saliva, la placa dental y la mucosa nasal. A nivel clínico, el Staphylococcus warneri está relacionado

\footnotetext{
* Especialista de Cirugía Ortopédica y Traumatología (COT).

** Residente de COT.

*** Médica Especialista Miembro de la Unidad de Enfermedades Infecciosas.

**** Especialista en Microbiología y Parasitología. Responsable de la Sección de Diagnóstico de las Infecciones Intraabdominales, Osteoarticulares y Cutáneas.

***** Jefe de Servicio de COT. Jefe de la Unidad de Rodilla.

****** Jefe Clínico de Servicio de COT. Jefe de la Unidad de Sépticos.
}

Dirección para correspondencia:

David Rodríguez Montserrat

C/ Balmes 423 6B, 08022,

Barcelona, España.

Tel: (0034) 626899870

E-mail: drodriguezmontserrat@gmail.com

Este artículo puede ser consultado en versión completa en http:// www.medigraphic.com/actaortopedica
ABSTRACT. Septic arthritis usually occurs as an acute joint process that can cause a rapid destruction of the cartilage, if the necessary therapeutic measures were not taken. Rarely, Staphylococcus warneri may be the cause of this pathology although due to its diagnostic difficulty we can make mistakes in its treatment. We present the case of a patient with septic arthritis of the knee by this germ and we intend to remark what are the diagnostic measures and recommendations to consider for this osteoarticular infection.

Key words: Septic arthritis, knee, diagnostic, infection.

con endocarditis valvular, bacteriemia, espondilodiscitis, meningitis en pacientes con derivación ventrículo-peritoneal e infección urinaria. También suele estar relacionado con infecciones de implantes protésicos, sobre todo catéteres. A nivel osteoarticular, se reconoce en la literatura ${ }^{1}$ publicada como un patógeno emergente, capaz de causar infecciones osteoarticulares graves, generalmente en asociación con la presencia de materiales de implante; pero, en ocasiones, se encuentra presente en infecciones sin implante y en pacientes considerados inmunocompetentes, como en el caso de artritis séptica de rodilla que presentamos.

Los estafilococos relacionados, con más frecuencia, con la artritis séptica son los Staphylococcus aureus y Staphylococcus epidermidis. Clínicamente, suele presentarse como monoartritis aguda que puede provocar una rápida destrucción articular, si no se toman las medidas terapéuticas necesarias. La artritis séptica sin implante por Staphylococcus warneri es una entidad muy poco frecuente y de gran dificultad diagnóstica, por tratarse de un saprófito de la piel que se encuentra en más de $50 \%$ de la población, pudiendo dar lugar a falsos diagnósticos de infección articular. El servicio de microbiología juega un papel crucial en el diagnóstico de este tipo de infecciones. 


\section{Caso clínico}

Presentamos el caso de un varón de 42 años con antecedentes de artritis gotosa de difícil control, tratada con antiinflamatorios no esteroideos (AINEs) por su reumatólogo, que consulta al Servicio de Urgencias, en dos ocasiones en la misma semana, por presentar dolor intenso e impotencia funcional en la rodilla derecha. Diez días antes, su reumatólogo le realizó una artrocentesis e infiltración de corticoide. En la primera visita a urgencias refería dolor e impotencia funcional en la rodilla derecha con derrame articular asociado. Se realizó una artrocentesis donde se obtuvo un líquido de aspecto amarillento, turbio, con una bioquímica: 5,450 leucocitos $/ \mathrm{mm}^{3}, 86 \%$ polimorfonucleares (PMN), linfocitos $14 \%$ y una glucosa de $64 \mathrm{mg} / \mathrm{dl}$. No se observaron microorganismos en la tinción de Gram. Se orientó como una recidiva de la artritis gotosa y fue derivado a su reumatólogo de referencia para completar el estudio. Fue visitado a los dos días, refiriendo haber presentado episodios de febrícula de hasta $37.5^{\circ} \mathrm{C}$ y acompañado de dolor intenso e impotencia funcional en rodilla derecha con derrame articular a tensión asociado. Ante el empeoramiento clínico, el paciente fue derivado nuevamente al Servicio de Urgencias. A la exploración física con tumefacción en la rodilla derecha, calor y dolor, sin enrojecimiento, pero con derrame articular a tensión. No hubo otras articulaciones involucradas. La temperatura corporal era de $35.8^{\circ} \mathrm{C}$. El resto del examen clínico no presentaba anomalías y no presentaba clínica de artritis en el resto de las articulaciones. No se hallaron tofos ni deformidades articulares residuales. También se descartaron otros focos sépticos, tanto pulmonar, urinario o abdominal. Se procedió al ingreso del paciente y se realizó una nueva artrocentesis que mostró un líquido de aspecto amarillento, turbio con 15,750 leucocitos $/ \mathrm{mm}^{3}, 85 \%$ PMN y una glucosa de $3 \mathrm{mg} / \mathrm{dl}$. En la tinción de Gram no se observaron microorganismos. El cultivo microbiológico fue negativo y la bioquímica del líquido mostró abundantes cristales de urato. En la analítica general de ingreso los resultados fueron los siguiente: leucocitos 13,500, hemoglobina (Hb) $11.3 \mathrm{~g} / \mathrm{dl}$, hematocrito (Hto) 31.5\%, 431,000 plaquetas $/ \mathrm{mm}^{3}$, velocidad de sedimentacion globular (VSG) $112 \mathrm{~mm}$, proteína C reactiva (PCR) 281.9 mg/l, hiperferritinemia compatible con anemia por inflamación/infección y ácido úrico en sangre de 8.6 $\mathrm{mg} / \mathrm{dl}$, se obtuvieron hemocultivos y urocultivos que siempre fueron negativos. La radiología simple de la rodilla no mostró lesiones óseas agudas. Durante el ingreso, se obtuvieron los resultados del cultivo de la artrocentesis realizada en la primera visita a urgencias, donde se aisló un estafilococo coagulasa negativo (S. warneri) sensible a oxacilina, levofloxacino, clindamicina, rifampicina, vancomicina y trimetroprim/sulfametoxazol. El paciente evolucionó a un empeoramiento clínico, con aumento de los signos flogóticos y disfunción de la rodilla derecha, aunque se mantuvo hemodinámicamente estable en todo momento. Se orientó como una artritis séptica en paciente con gota mal controlada. Se inició antibioterapia con cloxacilina $2 \mathrm{mg} / 4$ h y se programó desbridamiento quirúrgico. Se realizó artrotomía con desbridamiento y sinovectomía, manteniéndose la cobertura antibiótica pautada. Se obtuvieron muestras intraoperatorias para cultivo del tejido sinovial que resultaron negativas. No se apreció lesiones condrales intraoperatorias y las radiografías del postoperatorio inmediato mostraban unas interlíneas conservadas (Figuras 1 y 2 ).

Tras el desbridamiento, el paciente presentó una evolución favorable, sin objetivarse complicaciones postquirúrgicas. Analíticamente se observó un descenso progresivo de los reactantes de fase aguda (PCR 16 mg/l). Tras cumplir 15 días de tratamiento con cloxacilina endovenosa, se pasó a levofloxacino oral con buena tolerancia. El paciente mantuvo la antibioterapia oral por tres semanas y realizó
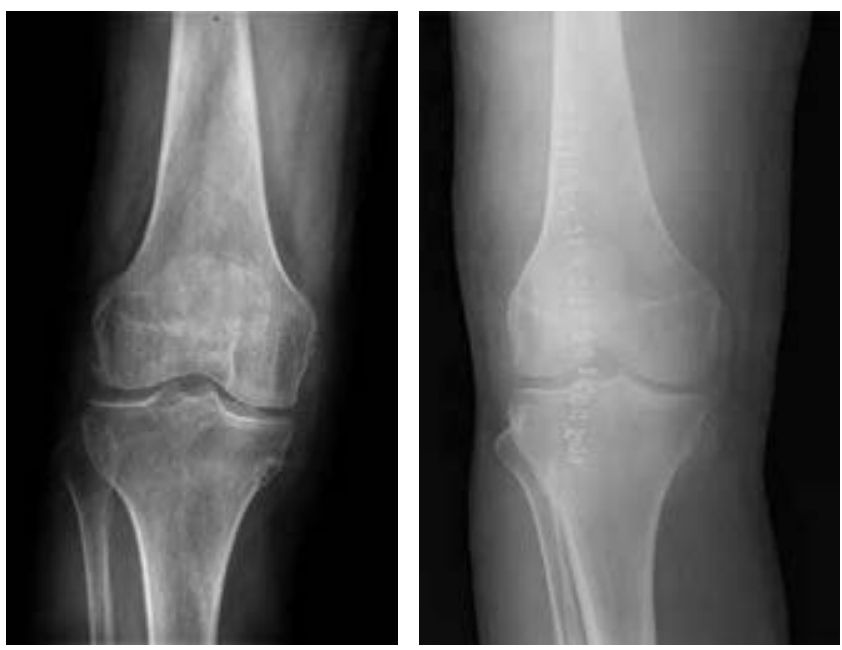

Figura 1: Radiografía anteroposterior (AP) bilateral postquirúrgica.

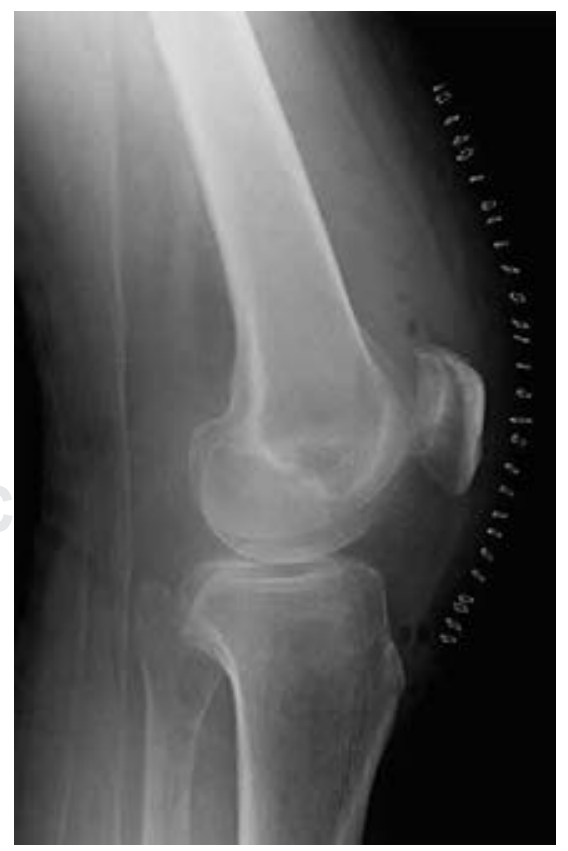

Figura 2: Radiografía lateral postquirúrgica. 
un protocolo de rehabilitación intensa recuperando parcialmente la atrofia muscular de cuádriceps con un resultado de fuerza final de $4+/ 5$. El balance articular inicial mostraba extensión de $10^{\circ}$ y una flexión de $60^{\circ}$ que después del tratamiento consiguió mejorar a $0-90^{\circ}$. Los valores de PCR al final del seguimiento tras parar el antibiótico volvieron al rango dentro de la normalidad (PCR $3 \mathrm{mg} / \mathrm{l}$ ). En la última visita de control presentaba un EVA 6/10 y continuaba realizando los ejercicios aprendidos durante el tratamiento de rehabilitación. El paciente es totalmente independiente para ABVD y deambula sin asistencia. A la exploración presentaba una correcta cicatrización de la herida con importante atrofia muscular en la extremidad y el estudio radiográfico al año de evolución no mostró afectación articular secundaria (Figura 3). La valoración objetiva de la funcionalidad del paciente, en fecha de última visita se realizó utilizando la escala WOMAC, obteniendo 7 puntos y la escala KSS (función) que fue de 168. Estos resultados objetivaron muy poca discapacidad funcional para las actividades de la vida diaria.

\section{Discusión}

Staphylococcus warneri representa $1 \%$ de todos los estafilococos. Aproximadamente, $50 \%$ de la población es portadora de esta especie a nivel cutáneo. Los casos de infecciones producidas por Staphylococcus warneri ${ }^{2}$ suelen estar relacionados con infecciones cutáneas, endocarditis sobre válvula protésica ${ }^{3}$ y espondilodiscitis. ${ }^{4}$ También se encuentra relacionado con infecciones neonatales, como los casos publicados por Cimiotti y colaboradores ${ }^{5}$ donde se describen bacteriemias, infecciones cutáneas y oculares producidas por este microorganismo. En la literatura, no quedan claros los factores de riesgo que se asocian a la infección por este microorganismo, pues en un número de casos no despreciable, se trata de infecciones adquiridas en la comunidad y en pacientes inmunocompetentes. También se ha relacionado con infecciones de implantes ortopédicos, tal
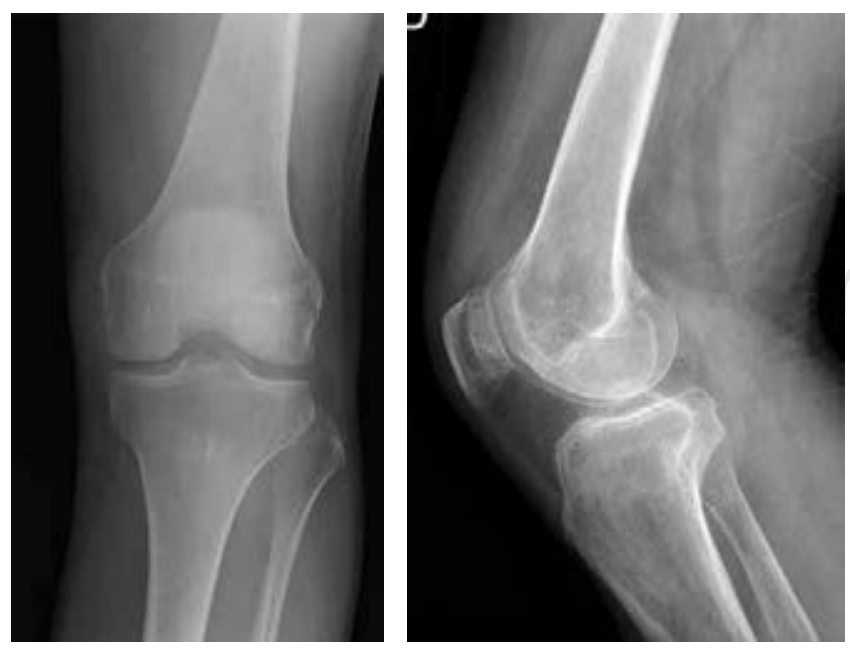

Figura 3: Radiografía anteroposterior (AP) y perfil al año de evolución. como se refleja en el trabajo de Arciola y asociados, ${ }^{6}$ donde Staphylococcus warneri se encuentra presente en $2.6 \%$ de los casos. Sin embargo, existen pocas series en la literatura, como el trabajo de Campoccia y su grupo, ${ }^{1}$ donde Staphylococcus warneri se identifica como microorganismo responsable de artritis séptica nativa, sin implante. Clínicamente, la artritis séptica por Staphylococcus warneri se presenta de forma muy similar a la producida por otras especies de estafilococos coagulasa negativa, como Staphylococcus epidermidis, Staphylococcus hominis o Staphylococcus capitis. Suele cursar sin fiebre o con febrícula y con menos signos flogóticos que las artritis sépticas producidas por otros microorganismos más virulentos como Staphylococcus aureus. En cuanto a la analítica, los parámetros de PCR y VSG en sangre, también suelen estar poco alterados, traduciendo una menor respuesta inflamatoria sistémica ante la agresión, Presentamos un caso de artritis séptica por Staphylococcus warneri en un paciente que, como peculiaridad, presentaba el antecedente de una artritis inflamatoria por depósito de cristales de base (ácido úrico) en la misma rodilla. Este hecho representa una dificultad añadida al diagnóstico. En estos casos, la anamnesis juega un papel importante en el diagnóstico, pues en muchas ocasiones va a ser difícil diferenciar si estamos ante un cuadro de reagudización de una artritis inflamatoria por cristales o por el contrario existe un episodio séptico concomitante. ${ }^{7}$ En nuestro caso, concretamente pensamos en la existencia de una posible complicación séptica debido al antecedente de infiltración articular previo al desarrollo del empeoramiento clínico de la artritis. Continuando con el diagnóstico, la artrocentesis y el estudio del líquido articular, tanto desde el punto de vista bioquímico como microbiológico, van a ser cruciales para el diagnóstico definitivo. La bioquímica del líquido articular, no presentaba un recuento absoluto de leucocitos tan elevado como para orientarlo como un proceso séptico o al menos no tanto, como el producido por Staphylococcus aureus, que suele cursar con más de 50,000 leucocitos $/ \mathrm{mm}^{3}$ en el líquido sinovial. ${ }^{8}$ Como hemos comentado anteriormente, este dato nos podría hacer pensar que estábamos ante un empeoramiento clínico de su artritis por cristales. Por otro lado, este recuento tampoco descartaría la existencia de una infección por alguna especie de estafilococo coagulasa negativa, entre ellos el Staphylococcus warneri, que puede cursar con recuento de leucocitos menores cuando provocan artritis sépticas. Como datos analíticos a favor de un proceso séptico concomitante, encontrábamos el elevado porcentaje de polimorfonucleares y el consumo de glucosa, con niveles menores del $50 \%$ de los encontrados en sangre. En general, en la artritis séptica, será el cultivo microbiológico el que nos aporte la confirmación diagnóstica, aunque debemos tener en cuenta que existe un porcentaje no despreciable de cultivos negativos. Según algunas series, este porcentaje llega a ser de $25 \%$, pudiéndose incrementar en los casos en los que el paciente ha sido tratado con antibiótico de manera empírica antes de la toma de muestras. ${ }^{9}$ Otro problema asociado al estudio microbiológico es que, cuando obtenemos 
un aislamiento de Staphylococcus warneri en un cultivo, va a ser difícil discernir si se trata de un contaminante o de un verdadero patógeno, debido a que es un microorganismo que puede formar parte de la flora comensal de la piel. Desde el punto de vista microbiológico y con el fin de optimizar el rendimiento de los cultivos, el líquido articular enviado en frascos de hemocultivo puede disminuir el tiempo de incubación, obteniendo de una manera más rápida el resultado microbiológico, ${ }^{10}$ tanto de la identificación del agente etiológico como del antibiograma. Esto permitirá ajustar y realizar un tratamiento antibiótico dirigido de una manera más precoz. Por todo ello, estamos ante un cuadro de artritis séptica, que, sin los resultados microbiológicos, resulta difícil de orientar como un cuadro de etiología infecciosa, debido al tipo de presentación clínica y a las escasas alteraciones analíticas que provoca, tanto en sangre como en líquido articular, si comparamos con otros tipos de microorganismos. La presencia de una artritis de cristales concomitante, además de un factor de confusión, se ha descrito en la literatura como un factor de mal pronóstico. Hunter y colaboradores ${ }^{11}$ encontraron que la presencia de una artritis inflamatoria concomitante a la artritis séptica aumentaba en siete veces el riesgo de fracaso del desbridamiento. En nuestro caso, el paciente presentó, tras un único desbridamiento con artrotomía y lavado-irrigación, una evolución favorable que, combinado con un tratamiento antibiótico adecuado, consiguió el control de la infección y una correcta recuperación funcional de la rodilla, sin llegar a presentar lesiones en el cartílago. En el tratamiento quirúrgico de la artritis aguda nativa sin implante, a diferencia de la infección protésica aguda, no parece existir diferencias en cuanto al control de la infección si realizamos un desbridamiento quirúrgico con artrotomía o realizamos una artroscopía. En esta línea, encontramos el trabajo prospectivo aleatorizado de Peres y asociados, ${ }^{12}$ donde la artrotomía y la artroscopía presentan tasas de control de infección similares, con una recuperación funcional más rápida y mejor control del dolor a favor de la artroscopía. Incluso hay autores como Bohler y su grupo que encuentran menos tasas de recidiva séptica cuando se realiza una artroscopía. ${ }^{13}$ Staphylococcus warneri es un estafilococo coagulasa negativo relacionado con frecuencia con la infección de implantes. Su diagnóstico puede ser difícil debido al crecimiento lento que posee y a su baja virulencia. Presentamos el caso de un paciente con una artritis séptica de rodilla por Staphylococcus warneri no asociada a implantes protésicos. A la dificultad propia del diagnóstico, en nuestro caso, se añade la existencia de una artritis gotosa concomitante, que puede actuar como factor de confusión y demorar el diagnóstico de artritis séptica. A pesar del lento crecimiento de este microorganismo y a diferencia de otros casos clínicos publicados, ${ }^{14}$ el diagnóstico lo realizamos mediante el estudio microbiológico del líquido articular conseguido por artrocentesis, utilizando los frascos de hemocultivos como medio de cultivo microbiológico. Además y como última reflexión, en pacientes con sospecha clínica de artritis séptica de rodilla no deberíamos iniciar el tratamiento antibiótico hasta la toma de muestras por artrocentesis, pues de esta manera se aumenta, en gran medida, el rendimiento de los cultivos microbiológicos.

Bibliografía

1. Campoccia D, Montanaro L, Visai L, Corazzari T, Poggio C, Pegreffi F, et al. Characterization of 26 Staphylococcus warneri isolates from orthopedic infections. Int J Artif Organs. 2010; 33(9): 575-81.

2. Wood CA. Significant infection caused by Staphylococcus warneri. $J$ Clin Microbiol. 1992; 30(8): 2216-7.

3. Arslan F, Saltoglu N, Mete B, Mert A. Recurrent Staphylococcus warneri prosthetic valve endocarditis: a case report and review. Ann Clin Microbiol Antimicrob. 2011; 10: 14.

4. Announ N, Mattei JP, Jaoua S, Fenollar F, Sati H, Chagnaud C, et al. Multifocal discitis caused by Staphylococcus warneri. Joint Bone Spine. 2004; 71(3): 240-2.

5. Cimiotti JP, Haas JP, Della-Latta P, Wu F, Saiman L, Larson EL. Prevalence and clinical relevance of Staphylococcus warneri in the neonatal intensive care unit. Infect Control Hosp Epidemiol. 2007; 28(3): 326-30.

6. Arciola CR, Campoccia D, An YH, Baldassarri L, Pirini V, Donati ME, et al. Prevalence and antibiotic resistance of 15 minor staphylococcal species colonizing orthopedic implants. Int J Artif Organs. 2006; 29(4): 395-401.

7. Yu KH, Luo SF, Liou LB, Wu YJ, Tsai WP, Chen JY, et al. Concomitant septic and gouty arthritis--an analysis of 30 cases. Rheumatology (Oxford). 2003; 42(9): 1062-6.

8. Liu NY, Giansiracusa DF. Septic arthritis. In: Gorbach SL, Bartlett JG, Blacklow NR, eds. Infectious disease. Philadelphia: WB Saunders; 1998: 1344-55.

9. Hindle P, Davidson E, Biant LC. Septic arthritis of the knee: the use and effect of antibiotics prior to diagnostic aspiration. Ann R Coll Surg Engl. 2012; 94(5): 351-5.

10. Font-Vizcarra L, García S, Martínez-Pastor JC, Sierra JM, Soriano A. Blood culture flasks for culturing synovial fluid in prosthetic joint infections. Clin Orthop Relat Res. 2010; 468(8): 2238-43.

11. Hunter JG, Gross JM, Dahl JD, Amsdell SL, Gorczyca JT. Risk factors for failure of a single surgical debridement in adults with acute septic arthritis. J Bone Joint Surg Am. 2015; 97(7): 558-64.

12. Peres LR, Marchitto RO, Pereira GS, Yoshino FS, de CastroFernandes M, Matsumoto MH. Arthrotomy versus arthroscopy in the treatment of septic arthritis of the knee in adults: a randomized clinical trial. Knee Surg Sports Traumatol Arthrosc. 2016; 24(10): 3155-62.

13. Böhler C, Dragana M, Puchner S, Windhager R, Holinka J. Treatment of septic arthritis of the knee: a comparison between arthroscopy and arthrotomy. Knee Surg Sports Traumatol Arthrosc. 2016; 24(10): 3147-54.

14. Legius B, Landuyt KV, Verschueren P, Westhovens R. Septic arthritis due to Staphylococcus warneri: a diagnostic challenge. Open Rheumatol J. 2012; 6: 310-1. 